\title{
Editorial
}

\section{Selected Papers from the 10th International Conference 2009 on Nonlinear Functional Analysis and Applications}

\author{
Yeol Je Cho' ${ }^{1}$ and Jong Kyu Kim ${ }^{2}$ \\ ${ }^{1}$ Department of Mathematics Education, Gyeongsang National University, \\ Chinju 660-701, Republic of Korea \\ ${ }^{2}$ Department of Mathematics Education, Kyungnam University, Changwon 631-701, Republic of Korea
}

Correspondence should be addressed to Yeol Je Cho, yjcho@gsnu.ac.kr and Jong Kyu Kim, jongkyuk@kyungnam.ac.kr

Received 30 December 2010; Accepted 30 December 2010

Copyright (c) 2010 Y. J. Cho and J. K. Kim. This is an open access article distributed under the Creative Commons Attribution License, which permits unrestricted use, distribution, and reproduction in any medium, provided the original work is properly cited.

During the last three decades, the problems of nonlinear analysis are a contemporary and very exhaustively studied research area and their applications can be found in functional analysis, fixed-point theory, variational inequalities, convex analysis, optimization, differential and integral equations, engineering, mathematical statistics, mathematical physics, astrophysics, and many other fields.

One of the most important and significant area is fixed-point theory being very rich, interesting, and extremely applicable areas of mathematics. The new results on fixedpoint theory are focused on the existence of fixed points of the classes of single-valued contractive, nonexpansive, accretive, monotone, fuzzy contractive mappings, and some kinds of multivalued mappings as well as extending the well-known results to more general spaces and applications in various areas of variational inequalities, complementarity problems, convex analysis, optimization, differential and integral equations, stability of functional, and applied sciences.

Inspired the importance of fixed-point theory and applications, since 1995, we have organized International Conference on Nonlinear Functional Analysis and Applications, which has been held at Gyeongsang National University, Chinju City, and Kyungnam University, Masan City, Korea, by the financial support of two universities, the Korean Mathematical Society, the Youngnam Mathematical Society, and the Korea Research Foundation and others. The purpose of this conference is to bring together leading researchers to achieve an international or higher level on the research of Nonlinear Functional Analysis with Applications and related topics and exchange recent new topics with each other. Especially, 
the 10th International Conference 2009 on Nonlinear Functional Analysis and Applications was held over the period July 27-31, 2009, at Gyengsang National University and Kyungnam University, Korea. Later, this conference will be held every two years.

Following these lines, this special issue of the Journal of Inequalities and Applications contains the selected 42 papers from the 10th International Conference 2009 on Nonlinear Functional Analysis and Applications as follows:

"Superstability and stability of the pexiderized multiplicative functional equations" by Y. W. Lee, "Fuzzy stability of an additive-quadratic-quartic functional equation" by C. Park, "Weak and strong convergence theorems for equilibrium problems and countable strict pseudocontractions in Hilbert Spaces" by R. Chen et al., "Strong convergence of an implicit iteration process for a finite family of uniformly L-Lipschitzian mappings in Banach spaces" by F. Gu, "Convergence theorems on generalized equilibrium problems and fixed point problems with applications" by Y. Hao et al., "A new iteration method for nonexpansive mappings and monotone mappings in Hilbert spaces" by J. S. Jung, "Approximation of solutions to a system of variational inclusions in Banach spaces" by X. Qin et al., "Moudafi's viscosity approximations with demi-continuous and strong pseudocontractions for nonexpansive semigroups" by $\mathrm{C}$. Wu et al., "Contiguous extensions of Dixon's theorem on the sum of a 3F2" by J. Choi, "Lyapunov inequalities for one-dimensional p-Laplacian problems with a singular weight function" by I. Sim, and Y. H. Lee, "Stability of quadratic functional equations via the fixed point and direct method" by E. Son et al. "Hyers-Ulam stability of differential equation $y^{\prime \prime}+2 x y^{\prime}-2 n y=0$ " by S. M. Jung, "Stability of a Cauchy-Jensen functional equation in quasiBanach spaces" by J. H. Bae and W.-G. Park, "An application of category theory to a class of systems of the superquadratic wave equations" by T. K. Jung and Q-H. Choi, "Modified block iterative algorithm for solving convex feasibility problems in Banach spaces" by S. S. Chang et al., "Controllability for variational inequalities of parabolic type with nonlinear perturbation" by J. M. Jeong et al., "Existence of solutions for $\eta$-generalized vector variational-like inequalities" by $\mathrm{X}$. Li et al., "Stability of approximate quadratic mappings" by H. M. Kim et al., "On the superstability of the pexider type trigonometric functional equations" by G. H. Kim, "An iterative algorithm of solution for quadratic minimization problem in Hilbert spaces" by L. Liu et al., "General iterative algorithm for nonexpansive semigroups and variational inequalities in Hilbert spaces" by $X$. Zhang et al., "A general iterative method of fixed points for mixed equilibrium problems and variational inclusion problems" by P. Katchang and P. Kumam, "Regularity for solutions of secondorder nonlinear integrodifferential functional equations" by D.-G. Park et al., "A hyperid iterative scheme for variational inequality problems for finite families of relatively weak quasi-nonexpansive mappings" by R. Wangkeeree and U. Kamraksa, "Optimality conditions and duality in nonsmooth multiobjective programs" by D. S. Kim and H. J. Lee, "Efficiency and generalized convex duality for nondifferentiable multiobjective programs" by D. S. Kim et al., "Optimality conditions in nondifferentiable G-invex multiobjective programming" by D. S. Kim et al., " $\epsilon$-duality theorems for convex semidefinite optimization problems with conic constraints" by G. M. Lee and J. H. Lee, "Implicit iteration methods with errors for discrete non-Lipschitzian families with perturbed mappings" by T.-H. Kim and K.-Y. Kim, "Strong convergence for generalized equilibrium problems, fixed point problems and relaxed cocoercive variational inequalities" by P. Kumam and C. Jaiboon, "Regularization inertial proximal point algorithm for monotone hemicontinuous mapping and inverse strongly monotone mapping in Hilbert spaces" by J. K. Kim and N. Buong, "Generalized biquasivariational inequalities for quasi-pseudomonotone type II operators on noncompact sets" by M. S. R. Chowdhury and Y. J. Cho, "Saddle point problems, bilevel problems, and mathematical programs with equilibrium constraints on complete metric spaces" by L. J. Lin and C.-S. Chuang, " $L^{2}$ error estimates of the extrapolated Crank-Nicolson discontinuous Galerkin approximations for nonlinear Sobolev equations" by M. R. Ohm et al., "Stability criterion for discrete-time systems" 
by K. Ratchagit and V. N. Phat, "Random stability of an additive-quadratic-quartic functional equation" by M. Mohamadi et al., "Convergence of iterative sequences for generalized equilibrium problems involving inverse-strongly monotone mappings" by S. M. Kang et al., "An iterative scheme with a countable family of nonexpansive mappings for variational inequality problems in Hilbert spaces" by Y. J. Cho and S. Wang, "Approximating curve and strong convergence of the CQ algorithm for the split feasibility problem" by F. Wang and $\mathrm{H}$. K. Xu, "New iterative schemes for asymptotically quasi-nonexpansive mappings" by Y. Yao and Y. C. Liou, "Some comparison inequalities for generalized Muirhead and identric means" by M.-K. Wang et al., "On the system of nonlinear mixed implicit equilibrium problems in Hilbert spaces" by Y. J. Cho and N. Petrot.

As guest editors for this special issue of Journal of Inequalities and Applications, we wish to thank all those who submitted manuscripts for publication and many specialists who served as the reviewers. We hope that all the papers published in this special issue can motivate and foster further scientific works and development that will allow for a continuous and better understanding of the role of nonlinear functional analysis and applications, especially, fixed-point theory, variational inequality problems, complementarity problems, equilibrium problems, convex analysis, minimization and optimization problems, stability of functional equations, differential and integral equations, inequality theory, KKM theory, and their applications.

\section{Acknowledgment}

Finally, we wish to thank Hindawi Publishing Corporation and Professor Ravi P. Agarwal, Editor-in-Chief of Journal of Inequalities and Applications for publishing Proceedings of the 10th International Conference 2009 on Nonlinear Functional Analysis and Applications as a special issue of Journal of Inequalities and Applications. 